\title{
Planning a high-frequency transfer-based bus network: How do we get there?
}

\author{
Emily Grisé \\ University of Alberta \\ egrise@ualberta.ca
}

\author{
Anson F. Stewart \\ Conveyal \\ astewart@conveyal.com
}

\author{
Ahmed El-Geneidy \\ McGill University \\ ahmed.elgeneidy@mcgill.ca
}

\begin{abstract}
As cities have grown more dispersed and auto-oriented, demand for travel has become increasingly difficult to meet via public transit. Public transit ridership, particularly bus ridership, has recently been on the decline in many urban areas in Canada and the United States, and many agencies have either undergone or are planning comprehensive bus network redesigns in response. While comprehensive bus network redesigns are not novel to public transit, network redesigns are commonly being considered in cities to optimize operational costs and reverse downward trends in transit ridership. For cities considering a comprehensive bus network redesign, there is currently no comprehensive easy-to-follow planning process available to guide cities through such a major undertaking. In light of that, this study presents a methodology to guide transport professionals through the planning process of a bus network redesign, using Longueuil, Quebec, as a case study. Currently, Longueuil operates a door-to-door network, and the goal is to move to a transfer-based, high-frequency service while keeping the existing number of buses constant. A variety of data sources that capture regional travel behavior and network performance are overlaid using a GIS-based grid-cell model to identify priority bus corridors. A series of analyses to measure and quantify anticipated and actual improvements from the proposed bus network redesign are conducted, including coverage analysis, change in accessibility to jobs, and travel time analysis. Accessibility to jobs was the key performance measure used in this analysis and is presented as a useful tool for planners and transit agencies to obtain buy-in for the proposed plan. This methodology provides transport professionals with a flexible and reproducible guide to consider when conducting a bus network redesign, while ensuring that such a network overhaul maximizes the number of opportunities that residents can access by transit and does not add an additional burden to an agency's operating budget.
\end{abstract}

Keywords: Public transport, bus network planning, bus network redesign, direct-service bus network

\section{Article history:}

Received: February 21, 2020

Received in revised form:

November 11, 2020

Accepted: March 11, 2021

Available online: August 13,

2021

Copyright 2021 Emily Grisé, Anson F. Stewart \& Ahmed El-Geneidy

http://dx.doi.org/10.5198/jtlu.2021.1742

ISSN: 1938-7849 | Licensed under the Creative Commons Attribution - Noncommercial License 4.0

The Journal of Transport and Land Use is the official journal of the World Society for Transport and Land Use (WSTLUR) and is published and sponsored by the University of Minnesota Center for Transportation Studies. 


\section{$1 \quad$ Introduction}

Public transit ridership has been on the decline in major North American regions (Boisjoly et al., 2018), and in 2018 bus ridership in the United States reached its lowest level since 1973 (National Academies of Sciences, 2020). In response, several cities have undertaken bus network redesigns, whereby transit agencies fundamentally alter the structure and organization of their bus network. Results of a survey of 36 cities in Canada and the United States whom either recently conducted a bus network redesign or are planning a redesign found that the national trend toward redesigns is motivated by three common themes (National Academies of Sciences, 2019). First, agencies have determined that existing route structure is not well aligned with present travel patterns and rider expectations. Second, agencies are determined to improve network efficiency. Third, in response to declining transit ridership in recent years, agencies are looking for strategies to reverse this trend and ideally boost ridership.

For transit systems to be aligned with existing and potential users expectations, a minimal level of service must be provided (Badia, Estrada, \& Robusté, 2016). While there is a common assertion that rail is inherently more attractive than bus, Ben-Akiva and Morikawa (2002) refuted this theory and found that high performance bus service with similar frequency and service attributes as rail (i.e., exclusive right-of-way) have similar ridership attraction. Service attributes associated with the common door-to-door bus network structure, or direct-service network, typically embody neither of these service attributes. A door-to-door bus network is designed to connect origin-destination pairs directly, with little need for transferring. Due to the existing sprawl in North American cities, providing door-to-door service requires circuitous routing, thereby extending travel times, and extensive coverage acts as a tradeoff for service frequency. Currently many cities are wishing to adopt a high-frequency transfer-based network, which have been demonstrated to improve operational efficiency and effectiveness (more users) in some North American cities (Brown \& Thompson, 2008; Thompson \& Matoff, 2003). In this paper, we present a practice-ready and easy to adopt methodology to guide transport professionals through the process of conducting a comprehensive bus network redesign, with the specific objective of transitioning a door-to-door bus network to a high-frequency transfer-based network, using Longueuil, Quebec, as a case study. The proposed bus network will maintain the number of buses in the existing network to ensure that the recommended network does not add an additional burden to the agency's operating budget.

A full network redesign traditionally progresses through three phases, which are iterative and may be performed in various sequences (National Academies of Sciences, 2019). Phase 1 involves gathering data (i.e., conduct a market analysis, establish or revise bus route and network service standards and budgetary/fleet resource limits), (2) Analyze and recommend (i.e., collect and analyze route and network performance data, consider service performance standards, and make recommendations for service changes), and (3) Engage the public (i.e., hold public engagements and revise recommendations based on public input). Our analysis exclusively focuses on the second phase of the bus service planning process, the recommendation of new bus routes, and recognizes that considerable additional work must also be conducted to successfully plan a network redesign.

\section{$2 \quad$ Background}

Transit network structure can be broadly categorized as either direct-service or transfer-based (Vuchic, 2005). Direct-service models, commonly referred to as a door-to-door model, connect suburban and inner-city areas with a central location, typically the downtown core. Typically, trips taken on a directservice model are designed so users can complete their trip using a single line. Routes in a direct-service 
network are predominantly designed in isolation of each other. Commuter trips are best served under this network model, providing efficient peak hour service to the CBD and sometimes other employment centers (Brown \& Thompson, 2008). Little or no transferring is required, which is a common motivation for adopting this network structure due to the common perception that transfers are disliked by passengers (Brown \& Thompson, 2008). A central issue with this service model, is that some users need to access destinations outside of the CBD, but using direct-service transit systems make this only possible with indirect travel through the CBD (Thompson \& Matoff, 2003). Furthermore, people need and want to travel at times outside of weekday peak hours.

Transfer-based networks are designed to allow riders to access multiple destinations when transfers are utilized. Common direct-service networks take on either a hybrid structure, which consists of a central grid network with radial routes serving suburban areas (see Daganzo, 2010), or a grid structure. Transfer-based networks are increasingly adopted with knowledge that destinations are increasingly dispersed in metropolitan areas (Brown \& Thompson, 2008), and in some settings this network model has been found to better satisfy dispersed mobility patterns (Badia et al., 2016; Daganzo, 1987; Thompson, 1977; Vuchic, 2005). Transit networks with many transfer opportunities offer passengers a significantly greater selection of travel paths compared to direct-service networks comprised of a large number of integrated lines that involve little or no transfers (Vuchic, 2005). For example, the centralized radial bus network in Tallahassee, Florida was converted to a decentralized, grid-like network to better serve Tallahassee's decentralized development patterns (Bhattacharya, Brown, Jaroszynski, \& Batuhan, 2014). Transferring, however, can interrupt travel and cause significant travel time delays when timed poorly. With careful planning, such as good station design, convenient and safe walking paths, and frequent service across all routes, the inconvenience associated with transferring is minimized and benefits associated with transferring can be realized.

Barcelona, Spain, in 2012 transitioned from a direct-service network to a transfer-based network, based on the proposed network design by Estrada, Roca-Riu, Badia, Robusté, and Daganzo (2011). The network design followed these three properties: full area coverage with transfers and non-circuitous routings, easy to understand network design (i.e., grid), and high frequency (average headway of $6.2 \mathrm{~min}$ utes) (Badia, Argote-Cabanero, \& Daganzo, 2017). Preliminary analyses of this network transformation found that demand is already rising, and this growth is supported by transfers, suggesting that users are not averse to transferring when using a well-connected, high-frequency network (ibid). Complimenting these findings, Allen, Muñoz, and Rosell (2019), analyzed user satisfaction levels over a three year period following the implementation of the transfer-based network, to better understand how users' perceive the network reform. The authors observed higher satisfaction levels among the new lines relative to the existing service, due to increased frequency and improved reliability, but also for the added access to opportunities provided by the new network. Furthermore, it was observed that transferring does not penalize user satisfaction, suggesting that the improved mobility offered by transfer-based networks can more effectively produce satisfied customers, despite the additional transfers occurred when travelling.

\section{Study area}

Réseau de transport de Longueuil (RTL) provides public transit services to the five suburban regions that comprise the Agglomération de Longueuil: Boucherville, Brossard, Saint-Bruno-de-Montarville, Longueuil, and Saint-Lambert. The service area is $309.6 \mathrm{~km}^{2}$ and serves a population of 427,050 residents and on average experiences 100,000 trips per weekday (RTL, 2017). Currently, 23\% of residents in Longueuil commute to work by public transit (Statistics Canada, 2016).

Longueuil is located on the south shore of Montreal, Quebec (see Figure 1), and a large portion of 
residents commute daily to work in Montreal. The RTL network can be classified as a door-to-door bus network (or direct-service network), consisting of radial routes that follow circuitous routing for collection/distribution of riders in the low-density suburban residential areas and is designed to transport passengers to Montreal either via Terminus de Longueuil, which is a subway station operated by the Société de transport de Montréal (STM) or directly to Terminus Centre-Ville in downtown Montreal via the Champlain Bridge, see Figure 2. Median all-day route headways, as shown in Figure 2, are varied across the network and are typically large due to the extensive network coverage and length of the network (790 km) (RTL, 2019).

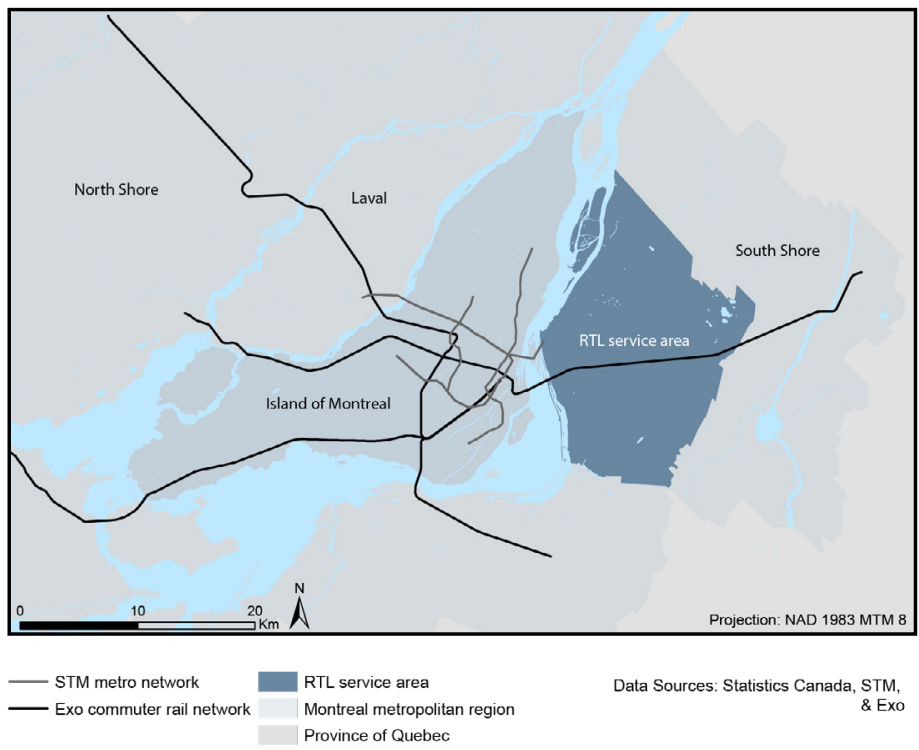

Figure 1. Context map of Montreal Metropolitan Region

In the Montreal Metropolitan region, the Réseau express métropolitain (REM) light-rail project is currently under construction. The REM will have implications for existing network design across the Montreal region. In the case of Longueuil, the REM will connect Longueuil to the Island of Montreal through Terminus Panama (Figure 2), and this service is projected to be operational in 2022. Currently, a large number of routes travel directly to Montreal via the Champlain Bridge, and this service will be replaced by the REM. To ensure a seamless transition of passengers to the REM, we are proposing an express bus service to connect passengers at Terminus Panama to downtown Montreal, which will be cancelled when the REM is operational. 


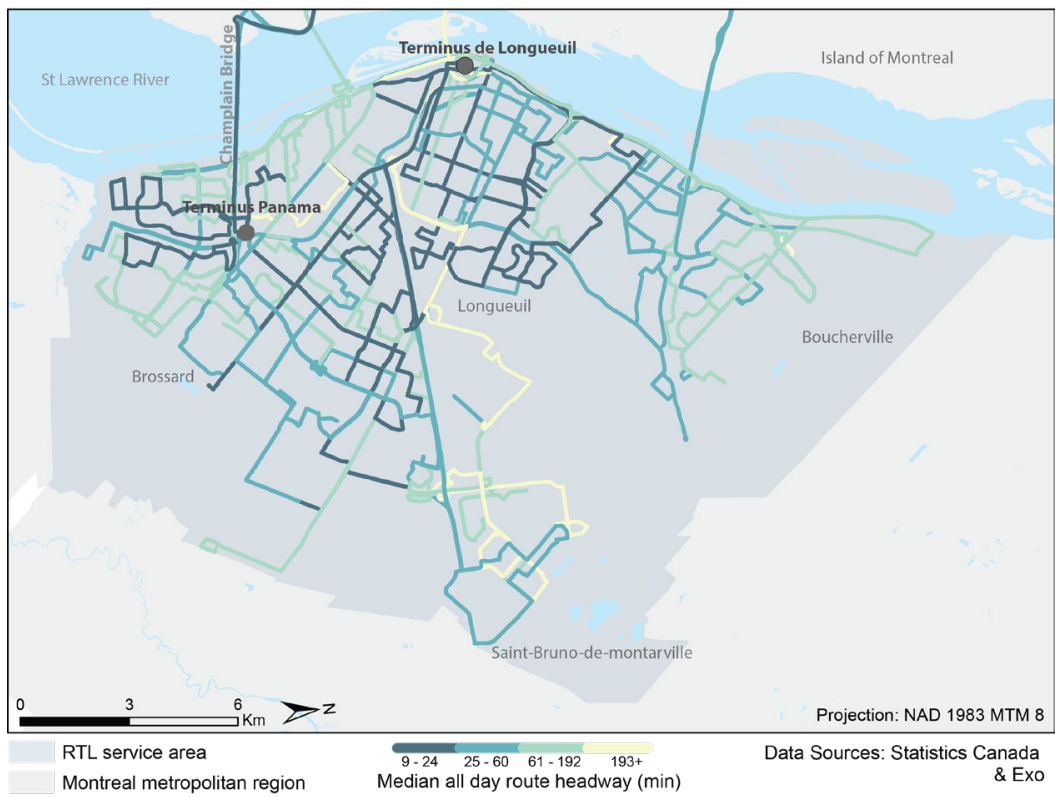

Figure 2. Existing door-to-door bus network, showing existing median all day route headways (minutes)

A challenge presented by this case study is the irregularity of Longueuil's street network. There is little pattern or order to streets in Longueuil. The most intuitive method of designing a transfer-based network is to follow a grid pattern, so that the network is easy to understand (Badia et al., 2017) and minimizes redundancies of service. That said, it is still very plausible to develop a transfer-based network in a city with an irregular street network, and in fact a grid-like network of north-south and east-west interconnected routes can still be developed.

\section{$4 \quad$ Methodology, application, and results}

The objective of this study is to develop a comprehensive methodology for transport engineers and planners to follow when conducting a bus network redesign. The specific goal of this network redesign is to transition an existing door-to-door bus network to a high-frequency transfer-based network. We outline below in detail the main steps deriving our proposed methodology for creating a new network and outline how this proposed network can be evaluated against the existing bus service. The approach taken is to devise a new network from scratch, rather than modifying the existing network, with the goal of identifying new patterns for service according to travel demand and land-use characteristics, while preserving strong features in the existing network. The authors recognize that there are several other approaches that could be taken when conducting a network redesign, including complex computational approaches to determining optimal network designs and optimization approaches that aim for minimal network change. Such a computational approach was found to be quite rare among cities that recently conducted a bus network redesign in Canada and the United States (National Academies of Sciences, 2019), in fact no cities used a pure computational approach among the surveyed cities. Several examples of transit network optimization approaches to network redesign do exist, for example the work of Badia, Estrada, and Robusté (2014) which was later implemented in Barcelona, Spain (Badia et al., 2017), and Lleida, Spain (Trapote-Barreira, Robusté, Badia-Rodríguez, \& Estrada-Romeu, 2016). Alternatively, Kalantari, Zamanian, and Amiripour (2014) presented a modification approach, whereby the authors proposed changes that optimize the performance of the existing network, and argue that this method is more ac- 
ceptable to riders and more likely to result in actual implementation when network changes are minor relative to a network designed with a blank slate. The method presented in this paper is designed to be implementable by both employees within transit agencies and consultants working with these agencies.

First, we develop a prioritization index using a Geographic Information System (GIS) grid-cell model to identify priority corridors for bus routes according to existing land-use characteristics, regional travel demand, and existing network performance. Second, we propose high and medium-priority bus routes, which is guided by the prioritization index. Third, we evaluate the coverage of the proposed network and measure changes in accessibility to jobs. According to these network evaluations, modifications to the proposed network were made. This process is completed iteratively, until we arrive at a new network that meets our two conditions: (i) offers sufficient network coverage to residents, and (ii) increase accessibility to jobs within 60 minutes by a minimum of 10 percent for all residents within the RTL service area. We complete our analysis by measuring whether the proposed service levels assumed in the accessibility analysis can be supported by the existing fleet. The existing fleet serves as a constraint in our analysis, whereby we are ensuring that the proposed network does not require the purchase of additional buses to deliver service.

\section{$5 \quad$ Prioritization index for new bus corridors}

To identify priority bus corridors, we consider a range of indicators that capture demand for travel in the Longueuil region and existing network performance and utilization. Specifically, seven indicators are considered in this analysis, which are described in detail below, and are each standardized and combined to a grid in GIS. This GIS-based grid cell prioritization model has been demonstrated in other applications, such as bicycle facility planning in Quebec City and Montreal, Canada (Grisé \& El-Geneidy, 2018; Larsen, Patterson, \& El-Geneidy, 2013). The seven indicators comprising this priority index were selected to capture existing and potential public transit demand and to help visualize optimal corridors.

\subsection{Land-use characteristics}

The first two indicators are employment and population density. Using data obtained from the Statistics Canada 2016 Census, employment and population density were calculated at the census tract level. These indicators ensure that proposed bus corridors are operating with high frequency in areas with high proportions of residents and jobs.

\subsection{Regional travel demand}

We then evaluated travel behavior in the region, using origin-destination (OD) survey data collected by the L'Autorité régionale de transport métropolitain (ARTM) in 2013 (Agence Métropolitaine de Transport, 2013). This data was used to generate desire lines, which represent demand for travel on each street segment. The OD survey collected by the ARTM surveys approximately $5 \%$ of the Montreal Metropolitan region and asks sampled participants over the phone to recall all trips made by themselves and other members of their household during the previous day. An expansion factor was applied to realistically capture the actual number of trips occurring in the region. Two indicators were generated, first an indicator representing all home-based trips for the purpose of work or school, and second all home-based trips by public transit. 


\subsection{Existing network performance}

The next three indicators we incorporated in our prioritization index were from automatic vehicle location (AVL) and automatic passenger counter (APC) data to assess existing network performance and demand. The three indicators include passenger activity, passenger load, and speed. AVL/APC data were provided by the RTL and included approximately two weeks of trip data in 2016 (December 5-18) and 2017 (September 4-17). We started by cleaning the data, to ensure that all records in our dataset were from trips operated by buses equipped with APC units, and we also removed incomplete trips from our sample. Our final sample consisted of weekday peak hour trips (6-9 AM and 4-6 PM) void of any holidays.

Passenger activity was calculated as the average number of boardings and alightings (ons and offs) occurring at each bus stop in the RTL network. In the case of a stop which serves multiple routes, passenger activity was calculated as the average number of boardings and alightings observed from all routes serving that stop (in both directions). This indicator was used to capture frequently used stops in the existing network to assign high priority to these locations for the future network.

Route passenger load was calculated using the maximum load field within the AVL/APC data, which indicates the highest load observed during a trip. Passenger load is the total number of onboard passengers at the time of departure from each stop and is used in this analysis to identify heavily used bus corridors. Load was calculated for each route in the network by obtaining the average maximum load of all trips in our data sample.

Our final network performance indicator is stop-level speed. Speed was calculated using travel time obtained from AVL/APC data, and distance travelled was obtained using GIS. A network of routes was generated in GIS by using the sequence of stops as recorded by AVL/APC data and stop coordinates provided by General Transit Feed Specification (GTFS). The stop coordinates were plotted in GIS and a network of routes was generated in Network Analyst ${ }^{1}$. Network distance between subsequent stops along each route was then obtained, and speeds then calculated using the average travel time between subsequent stops from the AVL/APC. Speed was incorporated in the prioritization index to identify low performance routes in terms of segment-level speed. The objective is to identify low performing routes where investments in priority bus corridors are needed, such as exclusive bus lanes, signal priority for buses or off-board payment methods to reduce dwell time associated with boarding passengers or adjusting stop locations (i.e., near-side versus far-side).

\subsection{Combining and spatially aggregating indicators to a grid}

Next, we aggregated the seven indicators to a grid cell with a $200 \mathrm{~m}$ resolution. For example, we spatially joined the passenger load indicator to the grid cells, to determine the average passenger load of routes intersecting each grid cell. Grid cells with high passenger load indicates areas where existing service is highly used and should be a priority for frequent bus service in the future network. In the case of the public transit commuting trips indicator, we measured the number of trips passing through each grid cell. Following the spatial aggregation of each indicator to the grid cell, we standardized each indicator (calculated a Z-score) to then add each indicator to the prioritization index. The seven standardized indicators are shown in Figure 3.

To combine all indicators into one priority index, we added each standardized indicator as follows:

\footnotetext{
1 We first snapped stop to streets, and then these stops were loaded into Network Analyst and grouped according to a route ID (route number and direction fields concatenated) and drawn according to their relative sequence along the route.
} 


\section{Prioritzation index}

$=$ Population density + Job density + Passenger activity

+ Passenger load + Flows all trips

+ Flows public transport trips - Speed

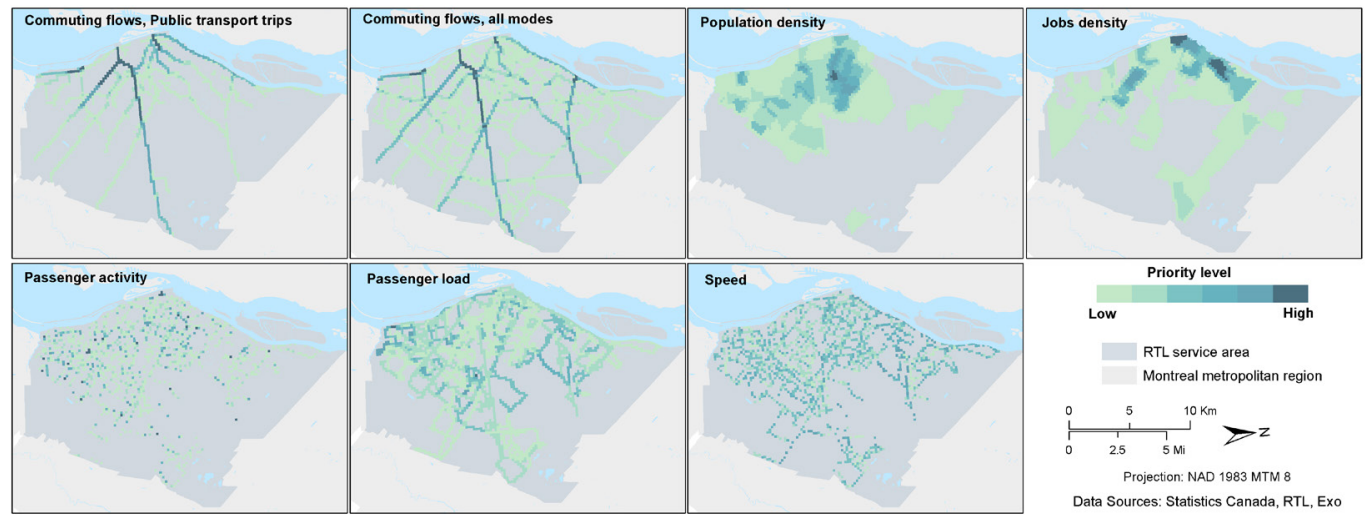

Figure 3. Standardized result of each indicator

Average speed is inversely added to our prioritization index as the goal of this analysis is to identify optimal corridors for high-frequency public transport services, whereby investments are made to improve the quality of service and reduce travel times. Note, we aggregated the seven indicators equally, and future applications of this method can consider applying a weighting scheme to prioritize certain indicators. The final prioritization index is presented in Figure 4.

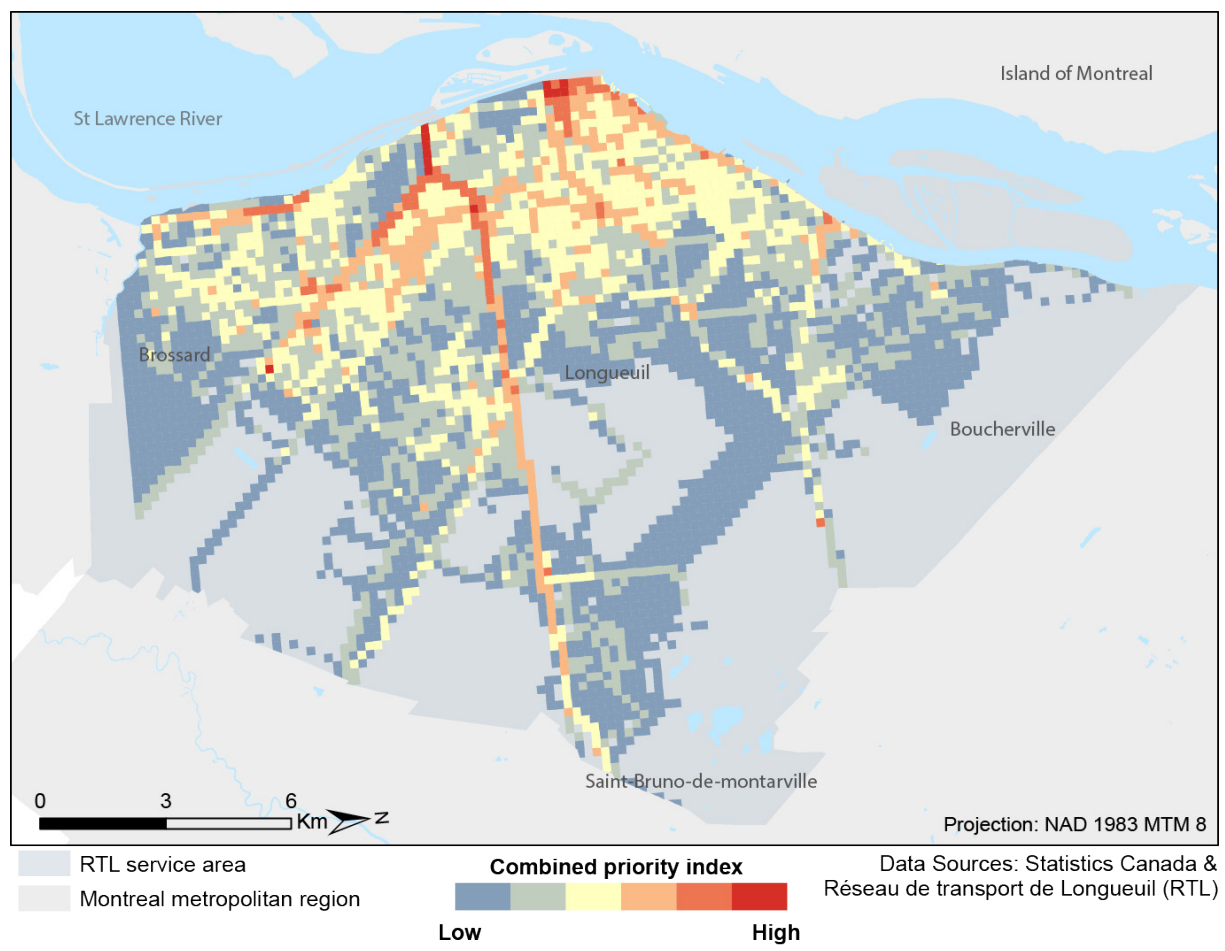

Figure 4. Final priority index 


\section{Proposed high and medium-priority bus routes}

Through close examination of the prioritization index (Figure 4), we identified several priority bus corridors that followed priority grid cells (red colored). Proposed routes will predominantly terminate at either Terminus Panama (future stop of the REM light rail service) or Terminus de Longueuil (subway station connecting riders to the Island of Montreal). At Terminus Panama passengers will transfer to an express downtown service to Terminus Centre-ville in downtown Montreal, which will be replaced by the REM service when it is completed. An average waiting time of 90 seconds is recommended for this route to minimize any inconvenience associated with transferring at this terminal. When drawing routes, we aimed to propose routes that follow major arterials and will flow in either a north-south or east-west direction to generate a grid. Also, when proposing routes along parallel corridors, whenever possible we attempted to place routes approximately 800 meters apart, so the maximum walking distance (laterally) is approximately 400 meters or 5 minutes. While shorter walking access is desirable, due to the importance of frequency for passenger attraction (Vuchic, 2005), we maximized frequency at the expense of access in some cases.

Our recommended high-priority routes, shown in pink in Figure 5, comprise the high-frequency routes forming the backbone of our network. These are predominantly radial routes, converging at either Terminus Longueuil or Panama. Our high-priority routes covered as many priority grid cells as

possible, however there are various barriers in the network that made this impossible, such as highways and railyards. These barriers also act as a hindrance from proposing a grid bus network. For example, there are limited locations for buses to travel east-west across the region, as many streets terminate on either side of the major north-south highway in Longueuil.

Next, we proposed medium-frequency bus routes (green routes shown in Figure 5) to integrate with the high-frequency routes and provide adequate coverage across the study region. When drawing these routes, we covered as many remaining high and medium-priority grid cells as possible, while also trying to optimize the location of parallel routes. Some of these medium-priority routes do not converge downtown and help comprise the grid transit network that we are aiming for, thereby allowing for more diverse travel patterns to be possible. Many of the proposed routes in the new network followed existing route alignments to utilize existing stops in the future, although in most cases we simplified routes, eliminating circuitous routing on local streets, to reduce travel time. 


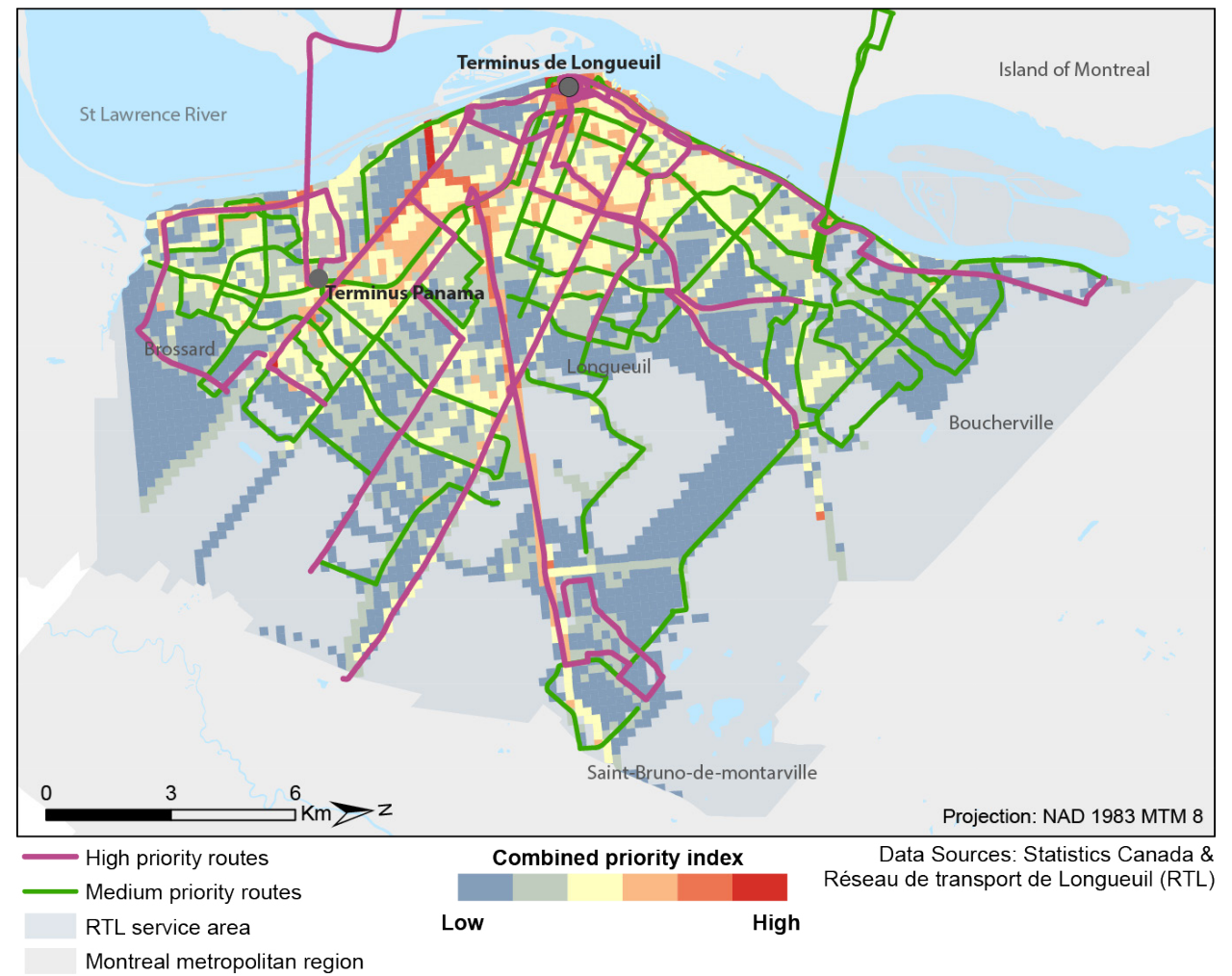

Figure 5. Proposed high- and medium-priority bus routes

\section{Coverage analysis}

To evaluate our proposed network, we first conducted a coverage analysis to identify network gaps that require improvements. This was done iteratively until a satisfactory level of coverage in the region was achieved, therefore meeting our first condition of a satisfactory network: 'Offers sufficient network coverage to residents'. To do so, we first measured the coverage of the existing network using 400m network buffers and then generated $600 \mathrm{~m}$ buffers around stops of the proposed bus network. A network buffer of $400 \mathrm{~m}$ is the most common standard measure of walking distance to bus stops (Gutiérrez \& GarcíaPalomares, 2008; Hsiao, Lu, Sterling, \& Weatherford, 1997; Kimpel, Dueker, \& El-Geneidy, 2007; O'Neill, Ramsey, \& Chou, 1992; Zhao, Chow, Li, Ubaka, \& Gan, 2003), although more recently studies have examined this buffer and found varying walking distances according to service type and frequency (El-Geneidy, Grimsrud, Wasfi, Tétreault, \& Surprenant-Legault, 2014). We adopted a 600m buffer for the proposed network as this is a high frequency network, and analyses of travel behavior have found that people walk further distances to routes with shorter waiting times (El-Geneidy et al., 2014).

Next, we compared the levels of coverage offered by the existing network and proposed network and applied modifications to the proposed network when major gaps were observed. As shown in Figure 6, we see minor losses of coverage within Longueuil. This sub-optimal spatial coverage was similarly observed in the case of Barcelona, due to a lack of suitability among select streets for operating highfrequency bus service (Estrada et al., 2011). Following close examination of these gaps in coverage, we determined that these were in suburban neighborhoods, with a striking lack of street connectivity and high proportion of cul-de-sacs. Due to the poor walkability of these neighborhoods, residents must walk 
further distances to access bus service on nearby arterials. To mitigate this, we suggest for planners to consider alternatives to connect theses residents to bus service, such as improved pedestrian connectivity to arterial streets or various services to help complete the first mile/last mile (e.g., transport network company (TNC) partnerships or on-demand bus service). Interestingly, there are also areas in the city where the proposed network offers more coverage than the existing one. What is most important to emphasize is the level of coverage achieved by the proposed network with the reduced kilometers of service proposed (790 km of existing service compared to $420 \mathrm{~km}$ of proposed service).

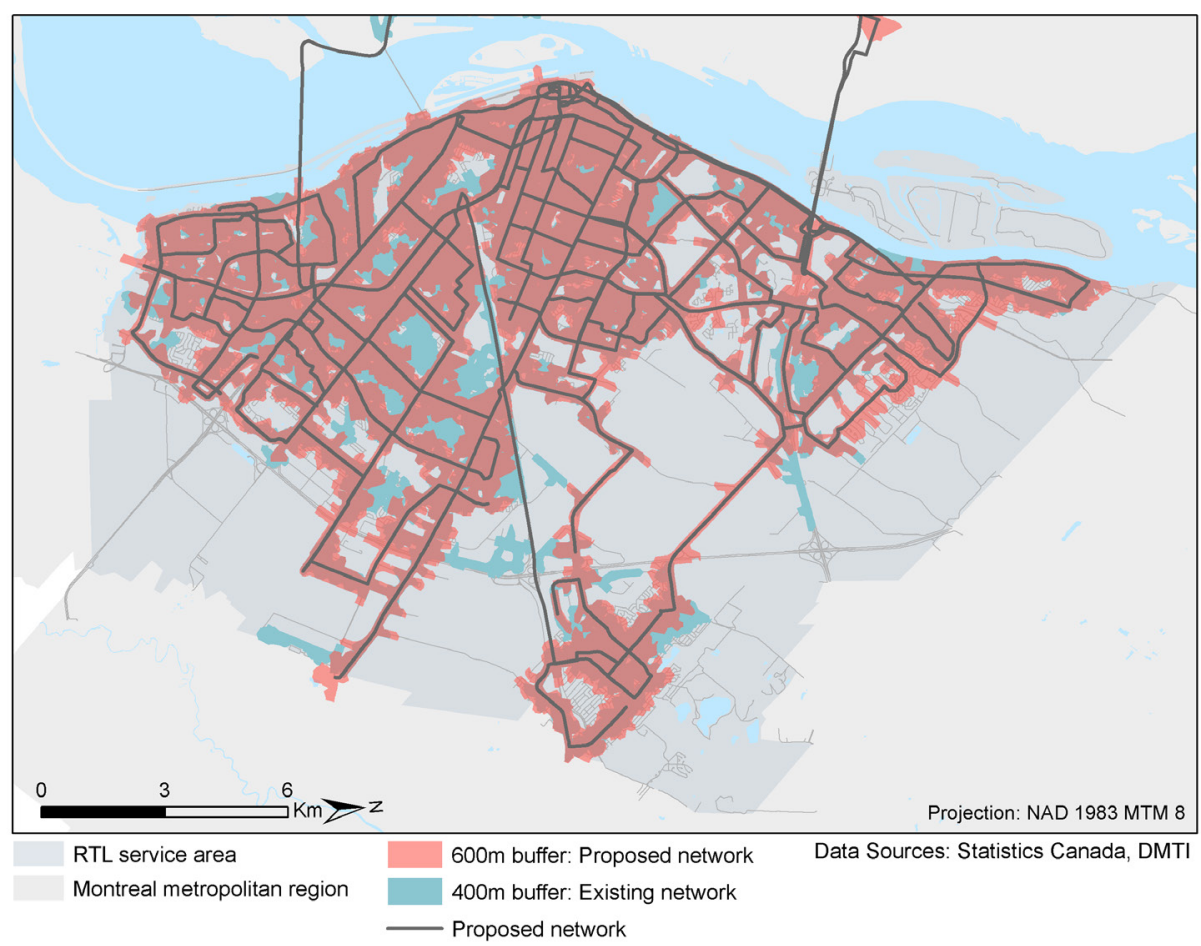

Figure 6. Comparing service coverage between the existing and proposed bus network

\section{$8 \quad$ Accessibility analysis}

The ultimate goal of this bus redesign is to provide existing users and potential users with improved access to desired opportunities locally and within the Montreal Metropolitan region. Accessibility is defined as a measure of potential opportunities (Hansen, 1959). The potential for reaching potential opportunities is determined by both the transport system (reflecting the travel time for reaching a destination) and the land-use system (Handy \& Niemeier, 1997). While reducing travel times is an important factor influencing passenger satisfaction levels (Dell'Olio, Ibeas, \& Cecin, 2011; Mouwen, 2015; Susilo $\&$ Cats, 2014), accessibility was selected as the focus of our network evaluation as it is essential that transport systems provides individuals with access to spatially and temporally dispersed opportunities (Banister, 2008; Straatemeier, 2008).

In this analysis, we measured accessibility to jobs, which is a commonly used proxy for density 
of activity in a region. A cumulative opportunity measure of accessibility of the existing and proposed network was calculated using Conveyal, an open-source cloud-based software. ${ }^{2}$ Jobs data was obtained from Statistics Canada 2016 Census Flow tables for the Montreal Metropolitan region, which represents the number commuters travelling between census tract (CT) pairs by mode of transport. Number of jobs is represented as the total number of commuters arriving per CT. Conveyal software measures accessibility at the grid cell level, with a resolution of $216 \mathrm{~m}$ by $216 \mathrm{~m}$ or approximately $0.05 \mathrm{~km}^{2}$, so number of jobs per CT was disaggregated by areal proportion into the grid cells intersecting each CT.

Travel time by public transit between all grid cells was determined using a 60-minute threshold, which was selected based on the existing travel behavior of residents. According to OD survey data, the median travel time of residents commuting by public transit is 55.9 minutes and the 75 th percentile travel time is 65.5 minutes. The resulting accessibility measures are presented in Figure 7. Travel time information for the existing network was obtained using GTFS data, and travel time was calculated for a generic Tuesday in May 2016. For the proposed network, GTFS data was drawn manually using Conveyal software's web-based interface ${ }^{3}$ using a route speed of $31 \mathrm{~km} / \mathrm{h}$, which is the median route speed of all scheduled daily trips in the GTFS feed of the existing network. Exceptions were made for routes that operate predominantly on highways with bus priority. The express route between downtown and Terminus Panama was assigned a speed of $35 \mathrm{~km} / \mathrm{h}$, and the remaining two express routes were assigned a speed of $45 \mathrm{~km} / \mathrm{h}$; both speeds closely resemble those of existing service on these corridors. When drawing routes, default stops were generated automatically at 500-meter increments, with subsequent manual adjustments to create convenient transfer points between routes and represent limitedstop express service. Finally, we assigned a headway to each route to generate timetables for all routes in our network. High priority routes were assigned an all-day headway of 7 minutes, medium priority routes were scheduled as a 10-minute headway throughout the day, and the express route to downtown was assigned a headway of 3 minutes. In the absence of a detailed proposed timeline for this network, Conveyal allows users to specify planned frequency of routes. While uncertainty in travel time will result in the absence of detailed timetables, this uncertainty can be visualized in the software. Uncertainty in travel time will be more significant for networks with less frequent service, and minimized when service across the network is frequent, such as the proposed network.

After calculating accessibility for each grid cell origin given the proposed and baseline networks, the two networks could be compared visually, highlighting remaining accessibility gaps to be addressed in additional iterations of network design. Several network iterations were carried out, where minor modifications to the proposed lines were made or new routes were added, until we reached a network satisfying our second condition: increase accessibility to jobs within 60 minutes by a minimum of 10 percent for all residents within the RTL service area.

Looking first at accessibility to jobs of the existing network, there are two hotspots of high accessibility, which are the two major transport terminals in Longueuil: Terminus Longueuil (subway station) and Terminus Panama (major bus terminal). In the remainder of the region, moderate and low levels of accessibility are seen. Looking at accessibility levels of the proposed network, we see that the proportion of grid cells with moderate and high levels of accessibility is strikingly higher, meaning that a much larger number of residents are residing in an area with good levels of accessibility to jobs relative to the existing network. We also see that levels of higher accessibility for the most part follow the proposed network, and therefore all residents living around the new network will experience good levels of accessibility.

\footnotetext{
2 Source code available at https://github.com/conveyal/analysis-backend

${ }^{3}$ Note, GTFS data of surrounding municipalities was used in this analysis, and existing RTL schedules were replaced with schedules of proposed network
} 
Looking closely at percentage change in accessibility (Figure 8) we see many areas of the region where significant gains in accessibility are experienced. There are also areas that experience declines in accessibility, which can be attributed to increased walking access to bus service in low density areas (identified in Figure 6). Also, moderate reductions in accessibility in the region of Brossard may be a result of the transfer required at Terminus Panama to the downtown-express bus service, whereas currently passengers can take a bus directly to the Island of Montreal.
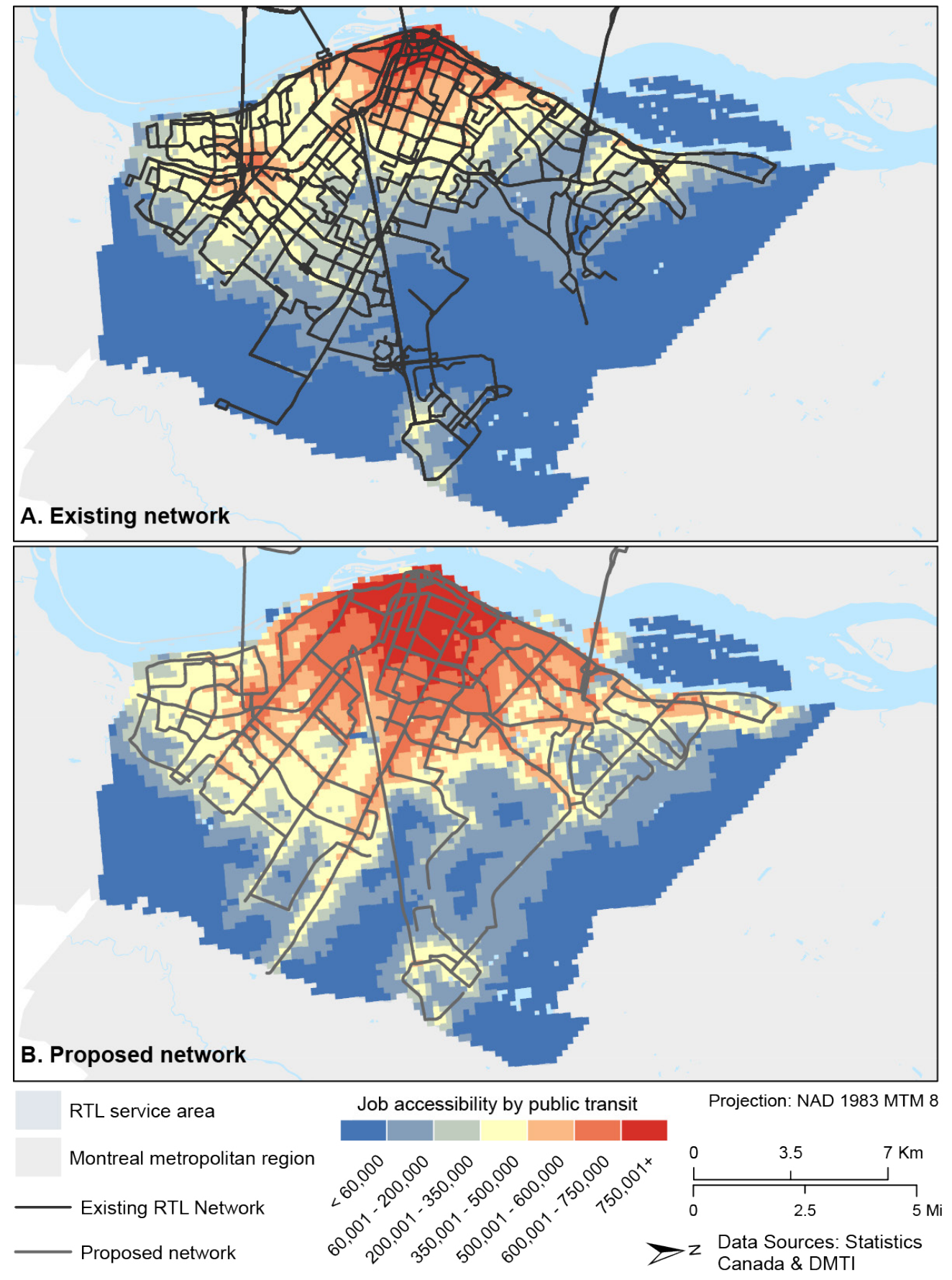

Figure 7. 60-minute accessibility to jobs (A) existing RTL bus network and (B) proposed bus network 
At a regional level, median accessibility to jobs within 60 minutes increases from 358,271 to 475,064 . This increase in job accessibility (approximately 33 percent) far exceeds our condition for an increase in accessibility of at least 10 percent. If the RTL were to instate a policy target that $75 \%$ of the service area's residents should be able to access at least $10 \%$ of the Montreal Metropolitan region's 1.75 million jobs, the redesign scenario would meet it: approximately $78 \%$ of the population has access to at least 175,000 jobs in the redesign scenario, versus only $68 \%$ in the baseline.

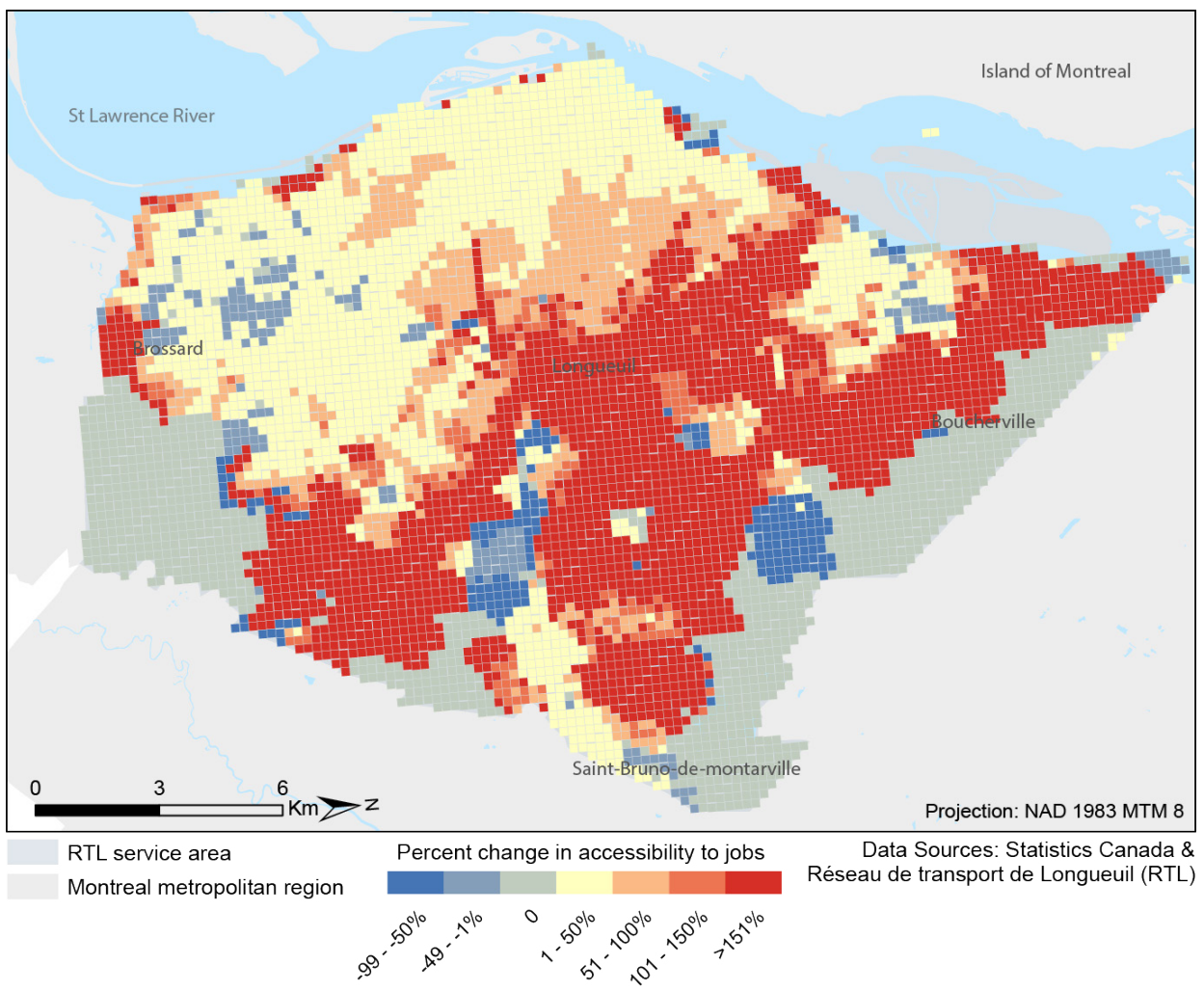

Figure 8. Percent change in accessibility to jobs

\section{$9 \quad$ Bus fleet analysis}

Following the proposal of a new bus network, we conducted a series of calculations to determine the number of buses required to operate the proposed network at peak service hours. This step was completed to ensure that the proposed network can be delivered using either the existing number of buses within the RTL fleet or with fewer buses to save operating costs. Currently, the RTL owns 484 buses, and we assumed that at any time a minimum of $10 \%$ of this fleet will be reserved for maintenance in the garage, leaving a maximum of 435 buses available to serve the network at peak.

We first calculated the cycle time for each route, which is the time for one vehicle to complete both directions of a route (out and return). Cycle time is expressed by the relationship:

Cycle Time $($ minutes $)=$ Travel Time $($ minutes $)+$ Layover (minutes $)$

Cycle Time (minutes) $=\frac{2^{*} \text { Length }(\mathrm{km})^{*} 60\left(\frac{\mathrm{min}}{\mathrm{hr}}\right)}{\text { Speed }(\mathrm{km} / \mathrm{h})}+$ LayoverTime 
In our calculations, we applied 40 minutes of layover (terminal) time, assuming a 10-minute break at one end and 30 at the other end. Finally, the number of vehicles required for each route is calculated as follows:

Number of Vehicles ${ }^{4}=\frac{\text { Cycle time (minutes) }}{\text { Headway (minutes) }}$

Using these formulas, we calculated the total number of buses required to operate the proposed network. Table 1 presents a scenario analysis, where we show total buses needed according to two levels of frequency, first a conservative scenario and a high-frequency scenario. We then show how operating speed changes the number of buses needed to serve each route. We begin with a speed of $31 \mathrm{~km} / \mathrm{h}$ as used in our accessibility analysis, and then present more conservative operating speeds: 28,25 and 22 $\mathrm{km} / \mathrm{h}$. Note, a constant speed of $35 \mathrm{~km} / \mathrm{h}$ for our express downtown route and $45 \mathrm{~km} / \mathrm{h}$ for the two remaining express routes were used.

Table 1. Bus fleet calculation

\begin{tabular}{|c|c|c|c|c|c|}
\hline \multicolumn{2}{|c|}{ Conservative frequency } & \multicolumn{4}{|c|}{ Operating speed } \\
\hline & Headway & $31 \mathrm{~km} / \mathrm{h}$ & $28 \mathrm{~km} / \mathrm{h}$ & $25 \mathrm{~km} / \mathrm{h}$ & $22 \mathrm{~km} / \mathrm{h}$ \\
\hline Express downtown & $3 \mathrm{~min}$ & 27 & 27 & 27 & 27 \\
\hline High priority routes & $7 \mathrm{~min}$ & 176 & 186 & 199 & 216 \\
\hline \multirow[t]{2}{*}{ Medium priority routes } & $10 \mathrm{~min}$ & 127 & 133 & 142 & 150 \\
\hline & Total buses needed: & 330 & 346 & 368 & 393 \\
\hline \multicolumn{2}{|c|}{ High frequency } & \multicolumn{4}{|c|}{ Operating speed } \\
\hline & Headway & $31 \mathrm{~km} / \mathrm{h}$ & $28 \mathrm{~km} / \mathrm{h}$ & $25 \mathrm{~km} / \mathrm{h}$ & $22 \mathrm{~km} / \mathrm{h}$ \\
\hline \multirow[t]{2}{*}{ Express downtown } & $2 \mathrm{~min}$ & 40 & 40 & 40 & \\
\hline & 40 & & & & \\
\hline High priority routes & $6 \min$ & 198 & 211 & 224 & 246 \\
\hline \multirow[t]{2}{*}{ Medium priority routes } & $9 \min$ & 148 & 155 & 164 & 176 \\
\hline & Total buses needed: & 386 & 406 & 428 & 462 \\
\hline
\end{tabular}

Under the conservative frequency scenario, we see that 330 buses are needed to serve the network assuming an average operating speed of $31 \mathrm{~km} / \mathrm{h}$, and as many as 393 buses are needed for network speeds averaging $22 \mathrm{~km} / \mathrm{h}$. Therefore, the conservative frequency scenario can be implemented with significant operational savings. Alternatively, the RTL can implement the high-frequency scenario, requiring 386 buses if average operating speeds are $31 \mathrm{~km} / \mathrm{h}$, or 406 buses for network speeds averaging $28 \mathrm{~km} / \mathrm{h}$ or 428 for speeds averaging $25 \mathrm{~km} / \mathrm{h}$. However, there would be an insufficient number of buses to serve the network if average operating speeds are as low as $22 \mathrm{~km} / \mathrm{h}$. This sensitivity analysis demonstrates that the proposed network is feasible from an operational standpoint or this network can save the agency operational costs which can be reinvested towards service quality improvements. Furthermore, this sensitivity analysis identifies the significant operational savings that can be realized by increasing average operating speed of bus service.

${ }^{4}$ Calculated number of vehicles is rounded up 


\section{Case study of individual travel time and accessibility changes}

As a final analysis of our proposed network (see Figure 9), we randomly selected 30 individuals from the 2013 OD survey (previously used to evaluate travel demand), and measured changes in travel time and accessibility to jobs for these individuals' commuting trips. Trips from selected OD pairs all originated within the RTL service area and each trip destination was located within the Montreal Metropolitan region. Our sample consists of trips by either transit or driving for the purpose of commuting to work or school. We observed that if the proposed network is implemented, these individuals would experience an average 13.9-minute reduction in their travel time (for their work based trip), or a 36\% travel time savings. These travel time reductions are quite significant and would likely have a positive effect on user satisfaction levels, as well as potentially attracting drivers to switch to public transit. Moreover, these individuals would benefit from an $83 \%$ increase in their accessibility to jobs relative to the existing network. This increase in accessibility can be attributed to both reduced travel time and the improved connections to varied destinations within the city due to the grid-like network. In future analyses, these travel time changes should be extended to the full sample of the OD survey.

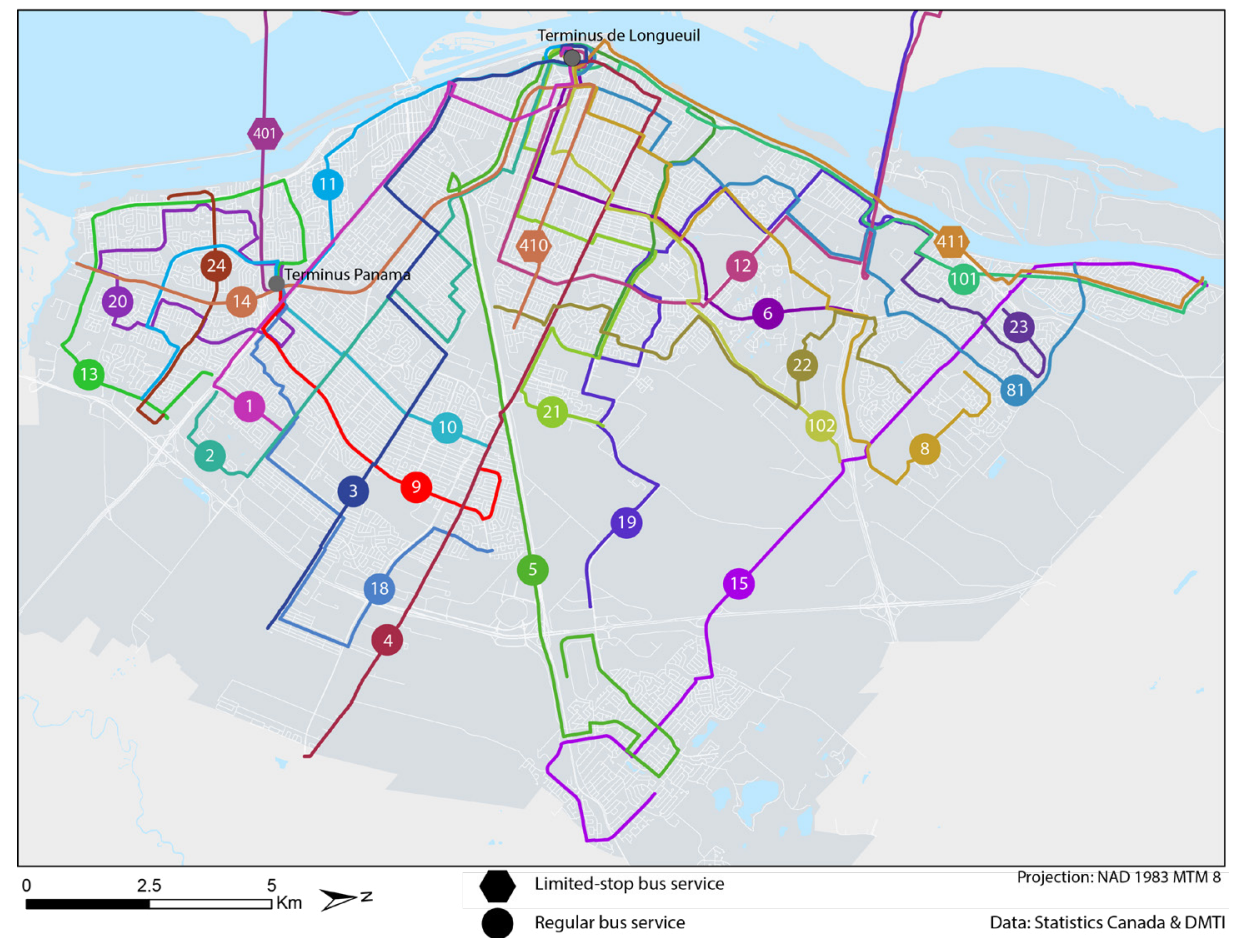

Figure 9. Final proposed network

\section{Conclusion}

For cities wishing to boost transit ridership and reduce single occupancy driving, it is essential to deliver quality transit service that is an attractive alternative to driving. Transport professionals have the knowledge that passengers desire frequent, reliable and fast transit service, and through comprehensive bus network redesigns public transit agencies can try and align service delivery with the expectations of existing and future customers. This study presents a methodology that can guide the process of transitioning a door-to-door bus network to a high-frequency transfer-based network. An overview of our 
methodology is shown in Figure 10. Using Longueuil, Quebec as a case study, we developed a method in GIS that consolidates multiple widely available datasets that describe the travel demand in the region, land-use characteristics, and existing network performance. A prioritization index was generated using these data to visually guide the proposal of high and medium-frequency bus routes.

In future applications of this methodology, inputs into the prioritization index can be changed according to data availability and a weighting scheme can be applied to place increased importance on certain indicators. Also, our study incorporated existing population and employment density and future applications of this methodology should consider future land-use changes, such as evaluating ongoing or proposed redevelopment in the city, and projected changes in travel demand as a result of shifting employment and residential density. For example, other approaches can apply 10- year projections of population and jobs to account for future demand as an indicator in the prioritization index. Furthermore, while we accounted for passenger loads and passenger demand in the prioritization index, our study should be followed by the generation of a demand model to estimate average hourly demand and to calculate the expected loads based on the new frequency. Based on the existing network demand (average all-day maximum route load is 11 passengers) we are not expecting imminent capacity issues in the new service.

While the street network in Longueuil lacks a clear pattern, we attempted to create a grid network to facilitate easy and intuitive transfers to spatially dispersed destinations in the city. The high-frequency routes that comprises the backbone of the network are predominantly radial routes, connecting to either Terminus Panama or Terminus Longueuil. Several medium priority routes do not travel to either terminal but instead help form the grid pattern, thereby connecting radial routes and enabling transfers.

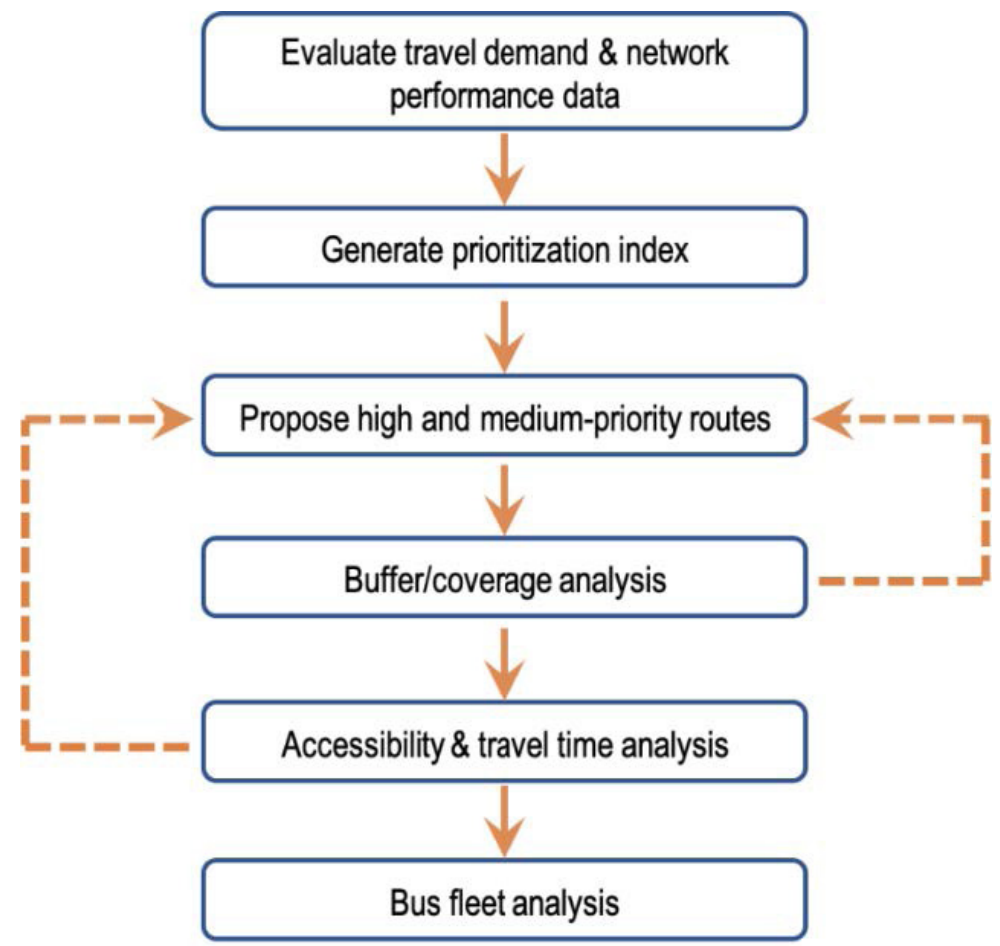

Figure 10. Overview of proposed methodology

Following the initial proposal of high and medium-priority routes, we evaluated the coverage of the routes and accessibility to jobs and modified the network accordingly. Several networks were proposed during our study, each improving on the network coverage or accessibility levels observed from previous 
network iterations. While minor losses in network coverage were observed throughout the region of Longueuil, in some parts of the city we achieved similar levels of coverage despite reducing the length of the network by approximately $47 \%$. Maximizing accessibility to jobs throughout the region was the ultimate target when evaluating the proposed network, rather than a mobility indicator such as travel time, since accessibility has been described as more accurately emphasizing the needs of individuals (Banister, 2008). While we did not plan for travel time, but accessibility, we noticed an average of 13.9 minutes of travel time savings, in other words travel time savings will come as a byproduct when planning for accessibility. At the regional level, median accessibility to jobs within one hour increases from 358,271 in the baseline scenario to 475,064 in the revised network. It is important to note that although the proposed network provides similar regional coverage and higher accessibility levels relative to the existing network, it requires significantly fewer buses than the RTL currently use in their fleet offering significant operational savings.

Changes in accessibility to jobs can be used to champion the proposed network. Transit accessibility is an intuitive and easily communicative measure of transit performance and can be used to communicate the benefits of the new network and generate buy-in of the network redesign from both the public and city officials as network changes are politically challenging for public transit agencies to implement.

Further evaluations should be considered in future analyses, including equity impacts, number of transfers (i.e., system-wide average transfer rate), and comprehensive travel time impacts (i.e., average transit travel time by census tract/neighborhood). Moreover, we recommend that planners apply an equity lens in future applications of this method by focusing on accessibility and travel time improvements in the most socially disadvantaged neighborhoods. Proactively considering disadvantaged neighborhoods throughout the planning process, through both public engagement and quantitative analyses like this proposed methodology, will have considerable benefit for these communities rather than evaluating how well a transport plan meets equity goals.

This study is intended to be easily replicated in other contexts by planners and engineers wishing to undertake a bus network redesign. This research illustrates the operational advantages of implementing a high-frequency transfer-based network, namely the reduced number of buses required to serve this network design relative to a door-to-door network. It is anticipated that implementation of this network structure will have positive impacts on ridership and satisfaction levels, as observed by Badia et al. (2017) and Allen et al. (2019) in the case of Barcelona's network reform. However, further research is needed to measure changes in ridership and satisfaction before and after comprehensive bus network redesigns, particularly in the North American context.

The expected opening of a new light rail project in the region offered a unique opportunity to rethink the entire RTL bus network. While such an opportunity does not happen every year for all agencies around the world, with the global prevalence of new rail projects or competing forms of transportation, this methodology can be of value to many practitioners who are similarly undergoing major network reforms. Alternatively, as cities grow and evolve, so do transit systems, often resulting in network inefficiencies or redundancies. We recommend transit agencies to conduct a network reform exercise such as this to determine whether the existing service is optimal for the region or whether network modifications should be proposed to improve service. A minimum average increase in accessibility of 10 percent is recommended as a measure to determine whether an agency should go forward with a network reform, thereby ensuring that such a network reform is a worthy investment. Furthermore, as network changes can be politically challenging to implement and can create significant concern and disruption from residents, it is critical that public transit agencies are presenting network changes that will result in notable benefits to everyone. Achieving substantial improvements in accessibility are expected to result in ridership gains (see previous work that has associated higher accessibility at transit 
stops with higher ridership (Dill, Schlossberg, Ma, \& Meyer, 2013)), as well as increases in transit mode share, as supported by several studies (Boisjoly \& El-Geneidy, 2016; Cui \& El-Geneidy, 2019; Owen \& Levinson, 2015).

\section{Acknowledgements}

We wish to thank Nicholas Tanguay, Michel Veilleux and Floriane Vayssieres from the Réseau de transport de Longueuil (RTL) for providing us with data and their input throughout this project. Note, the views of this paper do not reflect the opinions of the members of the RTL, rather this methodology was generated as an academic exercise. We would like to thank Dr. David Verbich from Stantec for inspiring us to write this paper. We also wish to thank Maaz Khan for his efforts with early stages of this project. This work was supported by research grants from the Natural Sciences and Engineering Research Council of Canada (NSERC) as well as the Social Sciences and Humanities Research Council (SSHRC). 


\section{References}

Agence Métropolitaine de Transport. (2013). Enquête origine-destination 2013. Montreal: Agence Métropolitaine de Transport.

Allen, J., Muñoz, J., \& Rosell, J. (2019). Effect of a major network reform on bus transit satisfaction. Transportation Research Part A: Policy and Practice, 124, 310-333.

Badia, H., Argote-Cabanero, J., \& Daganzo, C. (2017). How network structure can boost and shape the demand for bus transit. Transportation Research Part A: Policy and Practice, 103, 83-94.

Badia, H., Estrada, M., \& Robusté, F. (2014). Competitive transit network design in cities with radial street patterns. Transportation Research Part B: Methodological, 59, 161-181.

Badia, H., Estrada, M., \& Robusté, F. (2016). Bus network structure and mobility pattern: A monocentric analytical approach on a grid street layout. Transportation Research Part B: Methodological, 93, $37-56$.

Banister, D. (2008). The sustainable mobility paradigm. Transport Policy, 15(2), 73-80. http://ac.elscdn.com/S0967070X07000820/1-s2.0-S0967070X07000820-main.pdf?_tid=6cf470aa-187e11e6-9112-00000aab0f27\&acdnat=1463084375_fafb4bd6b84b7861ae745255d646ff71

Ben-Akiva, M., \& Morikawa, T. (2002). Comparing ridership attraction of rail and bus. Transport Policy, 9(2), 107-116.

Bhattacharya, T., Brown, J., Jaroszynski, M., \& Batuhan, T. (2014). The effects of perception vs. "reality" on travel behavior after a major transit service change: The case of Tallahassee, Florida. Journal of Public Transportation, 17(2), 1-26.

Boisjoly, G., \& El-Geneidy, A. (2016). Daily fluctuations in transit and job availability: A comparative assessment of time-sensitive accessibility measures. Journal of Transport Geography, 52, 73-81.

Boisjoly, G., Grisé, E., Maguire, M., Veillette, M., Deboosere, R., Berrebi, E., \& El-Geneidy, A. (2018). Invest in the ride: A 14 year longitudinal analysis of the determinants of public transport ridership in 25 North American cities. Transport Research Part A: Policy and Practice, 116, 434-445.

Brown, J., \& Thompson, G. (2008). Examining the influence of multidestination service orientation on transit service productivity: A multivariate analysis. Transportation, 35(2), 237-252.

Cui, B., \& El-Geneidy, A. (2019). Accessibility, equity, and mode share: A comparative analysis across 11 Canadian metropolitan areas. Transport Findings, February. https://doi.org/10.32866/7400

Daganzo, C. (1987). The break-bulk role of terminals in many-to-many logistic networks. Operations Research, 35(4), 543-555.

Daganzo, C. (2010). Structure of competitive transit networks. Transportation Research Part B: Methodological, 44(4), 434-446.

Dell'Olio, L., Ibeas, A., \& Cecin, P. (2011). The quality of service desired by public transport users. Transport Policy, 18(1), 217-227.

Dill, J., Schlossberg, M., Ma, L., \& Meyer, C. (2013). Predicting transit ridership at the stop level: The role of service and urban form. Paper presented at the 92nd annual meeting of the Transportation Research Board, Washington, DC.

El-Geneidy, A., Grimsrud, M., Wasfi, R., Tétreault, P., \& Surprenant-Legault, J. (2014). New evidence on walking distances to transit stops: Identifying redundancies and gaps using variable service areas. Transportation, 41(1), 193-210.

Estrada, M., Roca-Riu, M., Badia, H., Robusté, F., \& Daganzo, C. (2011). Design and implementation of efficient transit networks: Procedure, case study and validity test. Transportation Research Part A: Policy and Practice, 17, 113-135.

Grisé, E., \& El-Geneidy, A. (2018). If we build it, who will benefit? A multi-criteria approach for the prioritization of new bicycle lanes in Quebec City, Canada. Journal of Transport and Land Use, 
11(1), 217-235.

Gutiérrez, J., \& García-Palomares, J. (2008). Distance-measure impacts on the calculation of transport service areas using GIS. Environment and Planning B: Planning and Design, 35, 480-503.

Handy, S., \& Niemeier, D. (1997). Measuring accessibility: An exploration of issues and alternatives. Environment and planning A, 29(7), 1175-1194.

Hansen, W. (1959). How accessibility shapes land use. Journal of the American Institute of Planners, 25(2), 73-76.

Hsiao, S., Lu, J., Sterling, J., \& Weatherford, M. (1997). Use of geographic information system for analysis of transit pedestrian access. Transportation Research Record: Journal of the Transportation Research Board, 1604, 50-59.

Kalantari, N., Zamanian, M., \& Amiripour, S. (2014). Bus network modification problem: A new approach to bus network design. Paper presented at the Transportation Research Board, Washington, DC.

Kimpel, T., Dueker, K., \& El-Geneidy, A. (2007). Using GIS to measure the effect of overlapping service areas on passenger boardings at bus stops. Urban and Regional Information Systems Association Journal, 19(1), 5-11.

Larsen, J., Patterson, Z., \& El-Geneidy, A. (2013). Build it. But where? The use of geographic information systems in identifying locations for new cycling infrastructure. International Journal of Sustainable Transportation, 7(4), 299-317.

Mouwen, A. (2015). Drivers of customer satisfaction with public transport services. Transportation research Part A: Policy and Practice, 78, 1-20.

National Academies of Sciences, Engineering, and Medicine. (2019). Comprehensive bus network redesigns. Washington, DC: National Academies of Sciences, Engeneering, and Medicine.

National Academies of Sciences, Engineering, and Medicine. (2020). Analysis of recent public transit ridership trends. Washington, DC: National Academies of Sciences, Engineering, and Medicine.

O'Neill, W., Ramsey, R., \& Chou, J. (1992). Analysis of transit service areas using Geographic Information Systems. Transportation Research Record: Journal of the Transportation Research Board, 1364, 131-138.

Owen, A., \& Levinson, D. (2015). Modeling the commute mode share of transit using continuous accessibility to jobs. Transportation research Part A: Policy and Practice, 74, 110-122.

RTL. (2017). Annual report. Retrieved from http://www.rtl-longueuil.qc.ca/en-CA/press-room/documents-and-practical-information/annual-reports/

RTL. (2019). About. Retrieved from http://m.rtl-longueuil.qc.ca/en-CA/rtl/about

Statistics Canada. (2016). 2016 Census profile of census subdivisions. Retrieved from http://datacentre. chass.utoronto.ca/cgi-bin/census/2016/displayCensus.cgi?.year=2016\&geo=csd

Straatemeier, T. (2008). How to plan for regional accessibility? Transport Policy, 15(2), 127-137.

Susilo, Y., \& Cats, O. (2014). Exploring key determinants of travel satisfaction for multi-modal trips by different traveler groups. Transportation Research Part A: Policy and Practice, 67, 366-380.

Thompson, G. (1977). Planning considerations for alternative transit route structures. Journal of the American Planning Association, 43(2), 158-168.

Thompson, G., \& Matoff, T. (2003). Keeping up with Joneses: Radical vs. multidestinational transit in decentralizing regions. Journal of the American Planning Association, 69(3), 296.

Trapote-Barreira, C., Robusté, F., Badia-Rodríguez, H., \& Estrada-Romeu, M. (2016). Learnings from urban bus network change. Paper presented at the Transportation Research Board, Washington, DC.

Vuchic, V. (2005). Urban transit: Operations, planning, and economics. Hoboken, NJ: John Wiley \& Sons. 
Zhao, F., Chow, L., Li, M., Ubaka, I., \& Gan, A. (2003). Forecasting transit walk accessibility: Regression model alternative to buffer method. Transportation Research Record: Journal of the Transportation Research Board, 1835, 34-41. 